\title{
Alternating Coordinates Minimization Algorithm for Estimating Parameters of Partial Erasure Plus Transition Shift Model
}

\author{
Tsai-Sheng Kao and Mu-Huo Cheng, Member, IEEE
}

\begin{abstract}
The identification of model parameters of a highdensity recording channel is usually difficult and complicated. In this paper, we successfully apply the alternating coordinates minimization (ACM) algorithm for estimating parameters of a partial erasure plus transition shift model (PETSM). The resulting algorithm turns out to iteratively solve two least square problems and is guaranteed to converge. Furthermore, the obtained model for a nonlinear partial response channel is more accurate than conventional models such that the maximum likelihood (ML) detector has better bit error rate (BER) performance without increasing its realization complexity. Computer simulations show that the ACM algorithm can accurately estimate the model parameters and the BER for the detector is significantly improved especially when the transition shift parameter is large.
\end{abstract}

Index Terms-Alternating coordinates minimization, maximum likelihood detector, partial erasure ratio, partial response channel, transition shift parameter.

\section{INTRODUCTION}

$\mathbf{N}$ ONLINEAR distortions are the primary factors to limit the detector performance in high-density magnetic storage [1], [2]; these distortions are mainly the transition shift and the partial erasure. Several models have been presented to characterize the nonlinear distortions [3], [4], including the partial erasure plus transition shift model (PETSM) and simple partial erasure model (SPEM). However, the model parameters are usually difficult to estimate or measure [5], [6]. Recently, the authors applied the expectation-maximization (EM) algorithm [7] for identifying the parameters of a SPEM, and assumed that the effect of transition shift had been precompensated. This assumption makes the EM approach difficult to estimate model parameters directly from the measurement data without proper precompensation.

In this paper, the alternating coordinates minimization (ACM) algorithm [8], [9] is successfully applied for estimating parameters of a PETSM, including both nonlinear effects of transition shift and partial erasure. The resulting algorithm turns out to iteratively solve two least square problems and is guaranteed to converge. The obtained model for a nonlinear partial response channel is more accurate than conventional

Manuscript received October 15, 2003; revised December 17, 2003 and December 24, 2003. This work was supported by the National Science Council, Taiwan, under NSC92-2213-E-009-084.

The authors are with the Department of Electrical and Control Engineering, National Chiao Tung University, Hsinchu 300, Taiwan, R.O.C. (e-mail: mhcheng@cc.nctu.edu.tw).

Digital Object Identifier 10.1109/TMAG.2004.830223 models such that the maximum likelihood (ML) detector has better performance without increasing its realization complexity. The algorithm, therefore, enables us to accurately estimate parameters directly from the measurement data and to design a detector with improved performance.

\section{CHANNEL MODEL}

The sampled output, $y_{m}$, of the PETSM [4] is given by

$$
y_{m}=\sum_{k=-L_{1}}^{L_{2}} r_{m-k} b_{m-k} s\left(k T-\frac{b_{m-k} b_{m-k-1}}{4} \epsilon T\right)
$$

where $L_{1}, L_{2}$ express the duration of the channel, $s(t)$ is the Lorentzian function, $\epsilon$ is the transition shift parameter, $r_{m}$ represents the partial erasure effect, determined as follows:

$$
r_{m}= \begin{cases}1, & b_{m-1}=b_{m+1}=0 \\ \gamma_{1}, & \left|b_{m-1}\right| \neq\left|b_{m+1}\right| \\ \gamma_{2}, & \left|b_{m-1}\right|=\left|b_{m+1}\right|=2\end{cases}
$$

where $\gamma_{1}$ and $\gamma_{2}$ denote the partial erasure ratios. Note that the common setting for $\gamma_{2}=\gamma_{1}^{2}$ has been relaxed and thus the model flexibility is enhanced. The data $b_{m}$ is obtained by the nonreturn-to-zero-inverted (NRZI) encoding of the plus and minus binary recorded data $a_{m}$. Thus $b_{m}=a_{m}-a_{m-1}$ and $b_{m}$ may be of values $\{+2,0,-2\}$. Since the product $b_{m-k} b_{m-k-1}$ for all $k$ can only be either -4 or 0 , we denote a switch function $q_{m}$ by

$$
q_{m}=\left\{\begin{array}{ll}
1, & b_{m-1} b_{m}=0 \\
0, & b_{m-1} b_{m}=-4
\end{array} .\right.
$$

The PETSM (1) then can be represented in a new formulation

$$
y_{m}=\sum_{k=-L_{1}}^{L_{2}} r_{m-k} b_{m-k}\left[q_{m-k} g_{k}+\left(1-q_{m-k}\right) f_{k}\right]
$$

where the channel parameters $g_{k}=s(k T)$ and $f_{k}=s(k T+\epsilon T)$ for $k=-L_{1}, \ldots, 0, \ldots, L_{2}$. Therefore, the parameters of this model (4) consist of $\gamma_{1}, \gamma_{2}, g_{k}$, and $f_{k}$. The problem here is to find the model parameters for minimizing the following square output error

$$
J=\frac{1}{N} \sum_{m=1}^{N}\left(z_{m}-y_{m}\right)^{2}
$$

where $z_{m}$ is the sampled measurements of a magnetic recording channel and $N$ is the number of sample data. 


\section{ACM Algorithm fOR EStimating Model Parameters}

\section{A. Formulation of Two "Linear" Equations}

Divide the model parameters into two vectors $\boldsymbol{h}$ and $\boldsymbol{\lambda}$, with $\boldsymbol{h}$ representing the channel parameters and $\lambda$ representing the partial erasure ratio parameters, as follows:

$$
\begin{aligned}
\boldsymbol{h} & =\left[g_{-L_{1}}, f_{-L_{1}}, \ldots, g_{0}, f_{0}, \ldots, g_{L_{2}}, f_{L_{2}}\right]^{T} \\
\boldsymbol{\lambda} & =\left[\gamma_{1}, \gamma_{2}\right]^{T}
\end{aligned}
$$

where the superscript $T$ denotes the transpose operation. Denote $p_{m}=r_{m} b_{m}$ and

$$
\begin{gathered}
\boldsymbol{u}_{m}=\left[p_{m+L_{1}} q_{m+L_{1}}, p_{m+L_{1}}\left(1-q_{m+L_{1}}\right), \ldots, p_{m} q_{m},\right. \\
\left.p_{m}\left(1-q_{m}\right), \ldots, p_{m-L_{2}} q_{m-L_{2}}, p_{m-L_{2}}\left(1-q_{m-L_{2}}\right)\right]^{T}
\end{gathered}
$$

then the model output $y_{m}$ is a linear form of the channel parameters $\boldsymbol{h}$

$$
y_{m}=\boldsymbol{u}_{m}^{T} \boldsymbol{h} .
$$

Similarly, the model output also can be formulated as a linear form of the partial erasure ratio parameters $\boldsymbol{\lambda}$

$$
y_{m}=x_{m}+\boldsymbol{c}_{m}^{T} \boldsymbol{\lambda}
$$

where $\boldsymbol{c}_{m}=\left[\alpha_{m}, \beta_{m}\right]^{T}$, and

$$
\begin{aligned}
x_{m} & =\sum_{k=-L_{1}}^{L_{2}} b_{m-k} I_{m-k}\left[q_{m-k} g_{k}+\left(1-q_{m-k}\right) f_{k}\right], \\
I_{m-k} & = \begin{cases}1, & \text { if } b_{m-k-1}=b_{m-k+1}=0 \\
0, & \text { otherwise }\end{cases} \\
\alpha_{m} & =\sum_{k=-L_{1}}^{L_{2}} b_{m-k} I_{m-k}\left[q_{m-k} g_{k}+\left(1-q_{m-k}\right) f_{k}\right], \\
I_{m-k} & = \begin{cases}1, & \text { if }\left|b_{m-k-1}\right| \neq\left|b_{m-k+1}\right| \\
0, & \text { otherwise }\end{cases} \\
\beta_{m} & =\sum_{k=-L_{1}}^{L_{2}} b_{m-k} I_{m-k}\left[q_{m-k} g_{k}+\left(1-q_{m-k}\right) f_{k}\right], \\
I_{m-k} & = \begin{cases}1, & \text { if }\left|b_{m-k-1}\right|=\left|b_{m-k+1}\right|=2 . \\
0, & \text { otherwise }\end{cases}
\end{aligned}
$$

Note that (9) and (10) look like linear equations but in fact they are nonlinear.

\section{B. ACM Algorithm for Estimating Model Parameters}

The ACM algorithm iteratively performs the following two major operations until convergence; the iteration number is denoted by the subscript $k$. The first operation is given $\boldsymbol{h}=\boldsymbol{h}^{(k-1)}$ to solve $\lambda^{(k)}$ for minimizing $J$, which can be expressed as

$$
J(\boldsymbol{\lambda})=\frac{1}{N}\|z-\boldsymbol{x}-C \boldsymbol{\lambda}\|_{2}
$$

where $\boldsymbol{z}=\left[z_{1}, \ldots, z_{N}\right]^{T}, \boldsymbol{x}=\left[x_{1}, \ldots, x_{N}\right]^{T}$, and $C=\left[\boldsymbol{c}_{1}, \ldots, \boldsymbol{c}_{N}\right]^{T}$. Since the vector $\boldsymbol{x}$ and matrix $C$ are evaluated under the condition $\boldsymbol{h}=\boldsymbol{h}^{(k-1)}$, the performance $J$ in (14) is obviously a quadratic function of $\boldsymbol{\lambda}$ and the unique solution of $\boldsymbol{\lambda}^{(k)}$ can be obtained

$$
\boldsymbol{\lambda}^{(k)}=\left(C^{T} C\right)^{-1} C^{T}(z-x) .
$$

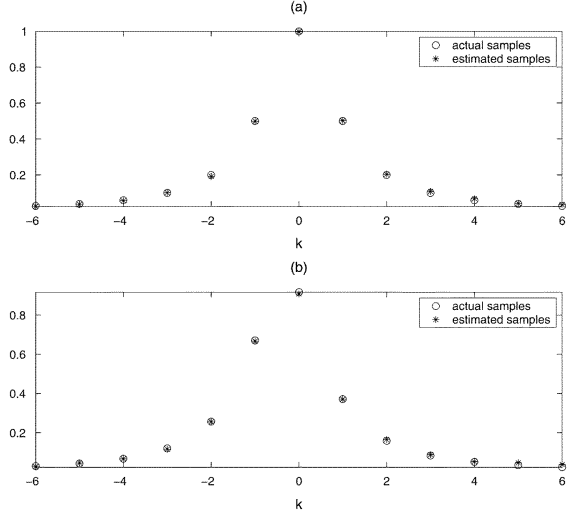

Fig. 1. (a) Samples of the channel parameters $g_{k} \mathrm{~s}$ and their estimates at the 257th iteration and (b) samples of the channel parameters $f_{k} \mathrm{~s}$ and their estimates at the 257 th iteration.

Similarly, the second operation is given $\boldsymbol{\lambda}=\boldsymbol{\lambda}^{(k)}$, obtained in the previous operation, to solve $\boldsymbol{h}^{(k)}$ for minimizing $J$, which is

$$
J(h)=\frac{1}{N}\|z-U h\|_{2}
$$

where $U=\left[\boldsymbol{u}_{1}, \ldots, \boldsymbol{u}_{N}\right]^{T}$, and each vector $\boldsymbol{u}_{m}$ is evaluated using (8) with $\lambda=\lambda^{(k)}$. The performance function $J$ in (16) is also quadratic and the solution is

$$
\boldsymbol{h}^{(k)}=\left(U^{T} U\right)^{-1} U^{T} \boldsymbol{z} .
$$

Each operation involves a quadratic minimization and solves a unique minimum. Thus, $J(\boldsymbol{\lambda}, \boldsymbol{h})$ is guaranteed nonincreasing. Furthermore, since $J(\boldsymbol{\lambda}, \boldsymbol{h})$ is bounded below by zero, the ACM algorithm will always converge. The algorithm here terminates when the measure, $\left\|\boldsymbol{h}^{(k)}-\boldsymbol{h}^{(k-1)}\right\|_{2}+\left\|\boldsymbol{\lambda}^{(k)}-\boldsymbol{\lambda}^{(k-1)}\right\|_{2}$, is less than a predetermined small value $\delta$.

\section{Simulation Example}

Let 1000 measurement data $z_{m}$ be generated as

$$
z_{m}=\sum_{k=-L_{1}}^{L_{2}} r_{m-k} b_{m-k} s\left(k T-\frac{b_{m-k} b_{m-k-1}}{4} \epsilon T\right)+n_{m}
$$

where $s(t)$ is also a Lorentzian function with $\mathrm{PW}_{50}=$ $2, \gamma_{1}=0.7, \gamma_{2}=0.49, \epsilon=0.3$, and $n_{m}$ is the additive white Gaussian noise. The noise variance is set to $-22.63 \mathrm{~dB}$ to make the signal-to-noise ratio (SNR), defined as $10 \log \left(\mathrm{E}\left[\left(z_{m}-n_{m}\right)^{2}\right] / \mathrm{E}\left[n_{m}^{2}\right]\right)$, equal $20 \mathrm{~dB}$. The channel lengths are given by $L_{1}=L_{2}=6$. The ACM algorithm is initialized with all model parameters set to zeros and the predetermined value $\delta=0.005$. Here, the algorithm terminated at the 257th iteration, and the convergent average square output error is $-22.51 \mathrm{~dB}$. The estimated partial erasure ratios $\hat{\gamma}_{1}$ and $\hat{\gamma}_{2}$ are respectively 0.7013 and 0.4942 , and the obtained model parameters are shown in Fig. 1, which illustrates that the ACM algorithm can accurately estimate the model parameters.

\section{NONLINEAR PR4 CHANNEL: Modeling And Detector Performance}

For high-density magnetic storage, the model duration is usually long; this makes the complexity to realize the detector, 
TABLE I

TOBTAINED SDRS IN dB OF EACH MODEL FOR PR4 CHANNEL WITH VARIOUS TRANSITION SHIFT VALUES

\begin{tabular}{c|c|c|c|c}
\hline$\epsilon$ & proposed model & Ryan's model & SPEM & LSM \\
\hline \hline 0.1 & 19.6836 & 19.1828 & 18.0882 & 9.3072 \\
0.2 & 18.9795 & 17.1686 & 14.9247 & 7.7017 \\
0.3 & 18.4324 & 14.5409 & 11.9430 & 5.9520 \\
0.4 & 17.9602 & 11.7578 & 9.5355 & 4.4162 \\
0.5 & 17.7718 & 9.5909 & 8.3392 & 3.8054 \\
\hline
\end{tabular}

designed for the obtained model, prohibitively high. Therefore, the magnetic channel is often equalized to a partial response model and then the ML detector is designed to improve the performance in high-density magnetic storage [10], [11]. Assume that the magnetic channel is equalized to the class-IV partial response (PR4) with minimum bandwidth [2], then its impulse response $s(t)$ is given by

$$
s(t)=\frac{\sin \frac{\pi t}{T}}{\frac{\pi t}{T}}+\frac{\sin \frac{\pi(t-T)}{T}}{\frac{\pi(t-T)}{T}} .
$$

If the nonlinear effects of partial erasure and transition shift are considered, the readback sampled signal, $z_{m}$, is obtained by

$$
z_{m}=\sum_{k=-L_{1}}^{L_{2}} r_{m-k} b_{m-k} s\left(k T-\frac{b_{m-k} b_{m-k-1}}{4} \epsilon T\right)+n_{m}
$$

where $L_{1}$ and $L_{2}$ represent the effective channel lengths of $s(t-$ $\epsilon T)$, and $n_{m}$ denotes the noise which is normally colored because of the PR4 equalizer. In this paper, the proposed model for the nonlinear PR4 channel is given by

$$
\begin{aligned}
y_{m}=r_{m} b_{m}\left[q_{m} g_{0}+\left(1-q_{m}\right) f_{0}\right] & \\
& +r_{m-1} b_{m-1}\left[q_{m-1} g_{1}+\left(1-q_{m-1}\right) f_{1}\right] .
\end{aligned}
$$

The parameters $\gamma_{1}, \gamma_{2}, g_{0}, f_{0}, g_{1}, f_{1}$ in (21) are further estimated by the ACM algorithm.

We use the signal-to-distortion (SDR) ratio, defined as $10 \log \left(\mathrm{E}\left[\left(z_{m}-n_{m}\right)^{2}\right] / \mathrm{E}\left[\left(z_{m}-y_{m}\right)^{2}\right]\right)$, to measure the model capability. The measurement data, for each value of $\epsilon$ from 0.1 to 0.5 , are generated using (20) with $L_{1}=L_{2}=10, \gamma_{1}=0.7$, and $\gamma_{2}=0.49$. For simplicity, white noise $n_{m}$ is used and the SNR is $20 \mathrm{~dB}$. The resulting SDRs are listed in Table I. The linear superposition model (LSM) results in the lowest SDR because it ignores the nonlinear effects. The SPEM [1] also yields poor performance in SDR when the transition shift parameter is equal to 0.2 or larger. While Ryan's model [2], because of linearization, produces high SDRs only for small $\epsilon$, our model (21) always results in a very high SDR even for $\epsilon$ as large as 0.5 .

The trellis diagram of the proposed model can be derived from (21) and is identical to those in [1] and [2], except that the model output is modified. The Viterbi algorithm is used to realize the ML detector for the detection of $b_{m}$. Since the data $b_{m}=a_{m}-a_{m-1}, a_{m}$ can be recovered by the relation $a_{m}=a_{m-1}$ when $b_{m}=0$ or $a_{m}=b_{m} / 2$ when $b_{m} \neq 0$. Note that recovering $a_{m}$ from $b_{m}$ may cause error propagation; this effect, however, is minor in our simulation. The bit error rate (BER) performance of $a_{m}$ for each model under various SNRs

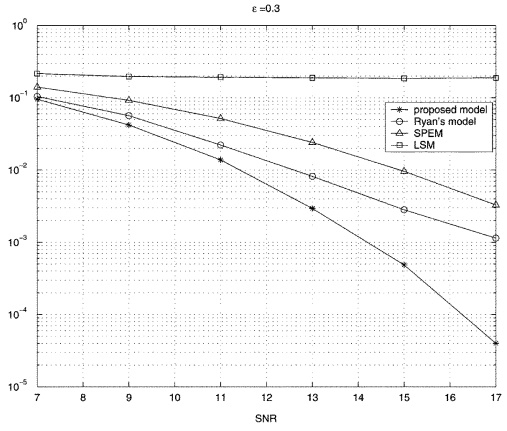

Fig. 2. Bit error rate of a nonlinear PR4 channel for $\epsilon=0.3$.

for $\epsilon=0.3$ is shown in Fig. 2. Hence, the complexity of the ML detector is not increased and the performance is improved because of the increasing model accuracy.

\section{CONCLUSION}

We have applied the alternating coordinates minimization algorithm for estimating the parameters of a PETSM. This algorithm can also be used to identify the parameters of the nonlinear PR4-equalized channel. The obtained model greatly increases the modeling accuracy and improves the performance of the corresponding ML detector without increasing its realization complexity.

\section{ACKNOWLEDGMENT}

The authors are grateful to the anonymous reviewers for their comments which greatly improved the quality of the paper.

\section{REFERENCES}

[1] I. Lee, T. Yamauchi, and J. M. Cioffi, "Modified maximum likelihood sequence estimation in a simple partial erasure model," in Proc. Int. Conf. Commun., 1994, pp. 245-249.

[2] W. E. Ryan and N. H. Yeh, "Viterbi detector for pr4-equalized magnetic recording channels with transition-shift and partial erasure nonlinearities," IEEE Trans. Magn., vol. 32, pp. 3950-3952, Sept. 1996.

[3] R. Hermann, "Volterra modeling of digital magnetic saturation recording channels," IEEE Trans. Magn., vol. 26, pp. 2125-2127, Sept. 1990.

[4] B. Lin, K. E. Hild, and J. R. Cruz, "Magnetoresistive read/write channel models," IEEE Trans. Magn., vol. 35, pp. 4528-4531, Nov. 1999.

[5] X. Che and P. A. Ziperovich, "A time-correlation method of calculating nonlinearities utilizing pseudo-random sequences," IEEE Trans. Magn., vol. 30, pp. 4239-4241, Nov. 1994.

[6] Y. S. Cho and N. J. Lee, "An estimation technique for nonlinear distortion in high-density magnetic recording channels," IEEE Trans. Magn., vol. 34, pp. 40-44, Jan. 1998.

[7] T. S. Kao and M. H. Cheng, "Expectation and maximization algorithm for estimating parameters of a simple partial erasure model," IEEE Trans. Magn., vol. 39, pp. 608-612, Jan. 2003.

[8] I. Csiszárr and G. Tusnády, "Information geometry and alternating minimization procedures," Statist. Decis., vol. supplement issue, pp. 205-237, 1984.

[9] A. Yeredor, "The extended least squares criterion: minimization algorithms and applications," IEEE Trans. Signal Processing, vol. 49, pp. 74-86, Jan. 2001.

[10] J. G. Proakis, "Equalization techniques for high-density magnetic recording," IEEE Signal Processing Mag., vol. 15, pp. 73-82, July 1998.

[11] R. W. Wood and D. A. Petersen, "Viterbi detection of class IV partial response on a magnetic recording channel," IEEE Trans. Magn., vol. 34, pp. 454-461, May 1986. 